\title{
Multicomponent exercise training in cardiovascular complexity in prefrail older adults: a randomized blinded clinical pilot study
}

\author{
M.S.S. Buto ${ }^{1 \oplus}$, V. Vassimon-Barroso ${ }^{1 \oplus}$, E. Fiogbé ${ }^{1 \oplus}$, A.C.S. Farche ${ }^{1 \oplus}$, B.F. Carnavale $^{1 \oplus}$ \\ P.G. Rossi ${ }^{1 \oplus}$, C.A. Sakaguchi ${ }^{1 \oplus}$, A.M. Catai ${ }^{1 \oplus}$, and A.C.M. Takahashi ${ }^{10 凶}$ \\ ${ }^{1}$ Departamento de Fisioterapia, Universidade Federal de São Carlos, São Carlos, SP, Brasil
}

\begin{abstract}
The aim of this study was to investigate the effects of multicomponent training on baroreflex sensitivity (BRS) and heart rate (HR) complexity of prefrail older adults. Twenty-one prefrail community-dwelling older adults were randomized and divided into multicomponent training intervention group (MulTI) and control group (CG). MulTI performed multicomponent exercise training over 16 weeks and CG was oriented to follow their own daily activities. The RR interval (RRi) and blood pressure (BP) series were recorded for $15 \mathrm{~min}$ in supine and $15 \mathrm{~min}$ in orthostatic positions, and calculation of BRS (phase, coherence, and gain) and HR complexity (sample entropy) were performed. A linear mixed model was applied for group, assessments, and their interaction effects in supine position. The same test was used to assess the active postural maneuver and it was applied separately to each group considering assessments (baseline and post-intervention) and positions (supine and orthostatic). The significance level established was $5 \%$. Cardiovascular control was impaired in prefrail older adults in supine position. Significant interactions were not observed between groups or assessments in terms of cardiovascular parameters. A 16-week multicomponent exercise training did not improve HR complexity or BRS in supine rest or in active postural maneuver in prefrail older adults.
\end{abstract}

Key words: Aging; Baroreflex; Complexity; Frailty; Physical exercise

\section{Introduction}

There is accumulating evidence that frailty may become one of the world's most serious health issues (1). Considering the expansive increase of the older adult population in the world, frailty prevalence tends to rise considerably (2) and consequently a burden on health and elderly care systems are also expected $(1,3)$.

In this context, frailty appears as one of the most problematic conditions, described as a clinical state of vulnerability to stress as a consequence of the decline of resilience and physiologic reserve related to aging, resulting in increased risk of adverse outcomes such as mortality, falls, institutionalization, hospitalization, loss of independence, and progressive decline in homeostasis (3-5).

The maintenance of homeostasis depends on a complex network of interactions among the control mechanisms. The aging process is accompanied by a reduction of these interactions in the physiological systems, which limits adequate response to stressors and characterizes the organism with reduced physiological complexity $(6,7)$. In frailty, a more pronounced loss of physiological complexity occurs, which would induce a loss of functional capacity to critical levels. Thus, the individual would become less resilient and therefore more vulnerable to development of pathologies and adverse outcomes as mentioned above (7).

Currently, the study of physiological complexity has been suggested in addition to traditional measurements in biological and health research (8). Once the physiological systems present a dynamic behavior, the complexity approach may offer an opportunity to characterize qualitatively these interactions (7), as well as baroreflex sensitivity (BRS) may represent interactions for blood pressure (BP) control. In this sense, one of the physiological systems most studied is the cardiovascular system $(7,9)$. It has already been demonstrated that frail older adults present impairment in cardiac complexity $(5,10,11)$ and in BRS (12).

Among the several interventions designed for frail and prefrail older adults, multicomponent exercise training has been demonstrated as the most effective for the reverse of

Correspondence: A.C.M. Takahashi: <anielle@ufscar.br>

Received September 14, 2020 | Accepted February 2, 2021 
frailty status and benefits in physical domains $(13,14)$. Nonetheless, the underlying physiological mechanisms remain unclear. To date there is little and divergent information about reversibility of cardiovascular complexity. Resistance training is effective for heart rate (HR) complexity improvement in young individuals as well as in hypertensive older adults $(15,16)$, but contrarily, it does not improve BRS in middle-aged people (17).

Thus, it remains unknown if an exercise intervention could promote benefits in cardiovascular complexity in prefrail elderly. In this sense, the aim of the present study was to verify if multicomponent exercise training could restore HR complexity as well as BRS in prefrail older adults.

\section{Material and Methods}

\section{Ethical approval}

This blinded randomized controlled trial was registered (Clinical Trial Registration ID: NCT03110419) and approved by the Research Ethics Committee of the institution (ID: 1800231/2016). Written consent was obtained from all the volunteers. All procedures were performed in accordance with the ethical standards of the 1964 Helsinki Declaration.

\section{Sample}

The inclusion criteria were community-dwelling prefrail older adults (according to frailty phenotype (4)) $\geqslant 65$ years old, with medical approval for exercising, and who agreed to participate in the study. The non-inclusion criteria were: a) Parkinson's disease; b) stroke; c) diabetes mellitus with peripheral neuropathy (18) assessed by Semmes-Weinstein monofilaments at 5.07 (10 g) (19); d) vestibular and visual self-reported disorders that would impair performance in assessment and/or training; e) an indication of cognitive deficit, assessed by means of the Mini Mental State Examination (MMSE) with scores lower than 18 (4); f) cardiovascular alterations (atrial fibrillation, malignant ventricular arrhythmia, complex ectopic ventricular beat, sinus or supraventricular tachycardia, second and third degree atrioventricular block; g) use of a pacemaker on resting electrocardiogram (ECG); h) unstable angina; and i) myocardial infarction.

Figure 1 shows a flowchart of the sample. Initially, 186 older adults were contacted. Forty-seven were not included due to age, comorbidities according to criteria, and MMSE score $<18$. Frailty screening was applied in 139 individuals and 99 were excluded due frailty status or non-interest in the study. Finally, 40 were considered eligible and were randomized into two groups of 20 subjects: i) the multicomponent training intervention group (MulTI), which participated in a multicomponent physical exercise protocol and ii) the control group (CG), which was oriented to follow their own habitual daily activities. After participation withdrawals and removal of signal artefacts, the final sample was composed by 21 subjects: MulTI $(n=12)$ and CG $(n=9)$ (see Figure 1).

\section{Study protocol}

Randomization process. After the first assessment, the participants were randomly distributed using the Random Allocation Software (Microsoft Corporation, USA)

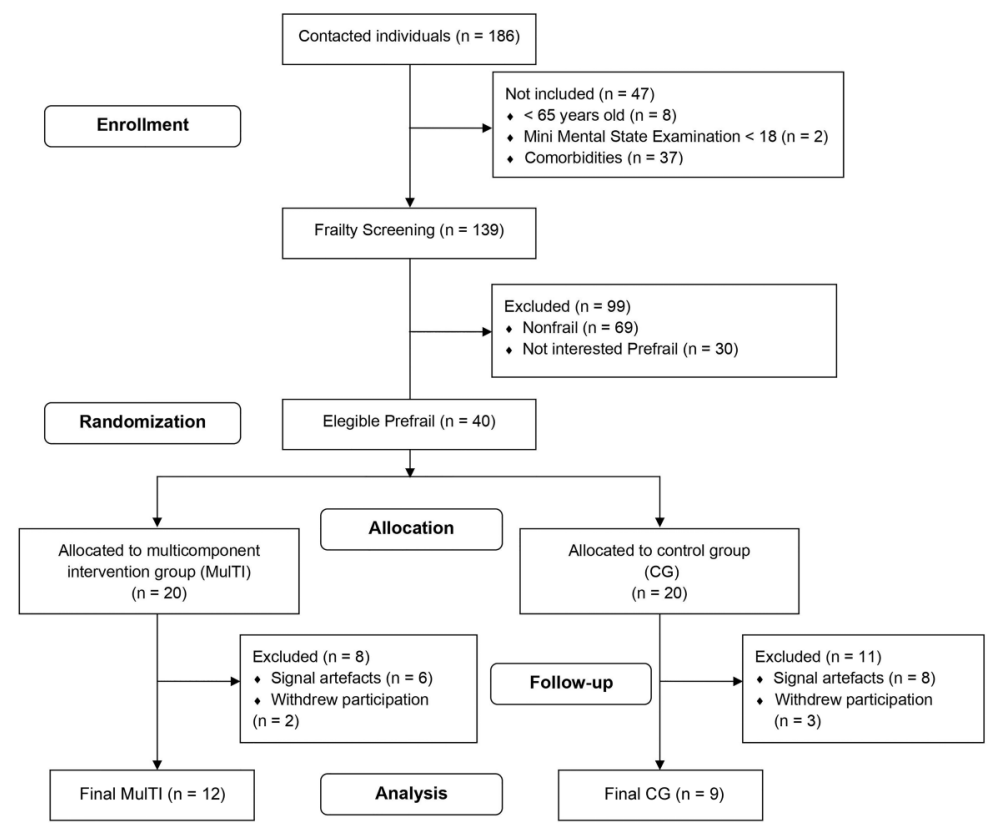

Figure 1. Flowchart of the final sample. 
into blocks of eight subjects. According to the randomization sequence, each participant was allocated according to a numbered card sealed in an opaque envelope indicating which group the individual would be inserted (MulTI or CG). The entire randomization process was performed by a researcher who had no link to the study (J.H.A.). The envelopes were opened after the first evaluation and the researchers were blinded about the allocation of the participants.

The assessments were performed at two distinct times: 1) baseline (initial assessment) and post-intervention (immediately after the conclusion of the 16-week multicomponent intervention).

Anamnesis. All participants were submitted to a structured interview. Demographic data (age and sex), educational level, MMSE total score, Short Physical Performance Battery (SPPB) total score, and comorbidities data were collected.

Procedures and experimental protocol. Initially, the volunteers were instructed to remain in supine position for $10 \mathrm{~min}$ in order to stabilize cardiovascular variables and to conduct the calibration procedure. ECG, blood pressure (BP), and breathing recordings were collected for $15 \mathrm{~min}$ in supine position. Then, the volunteers were instructed to actively change to orthostatic position, in which they remained for $15 \mathrm{~min}$. Previous instructions were given related to not ingest caffeine or alcohol or perform moderate or heavy exercise on the day before participation and to have a regular meal.

The experiments were conducted in a climatecontrolled $\left(22-23^{\circ} \mathrm{C}\right)$ room with relative air humidity of $40-60 \%$ always in the morning in order to minimize circadian cycle effects.

Signal acquisition. The ECG signal was collected by a bioamplifier (BioAmp Power Lab, Ad Instruments, Australia) with electrodes placed on the MC5 lead, and respiratory movements were captured by a respiratory belt (Marazza, Italy). The arterial BP waves were obtained by a plethysmography arterial pressure device (Finometer PRO, Finapress Medical Systems, The Netherlands), with a cuff placed on the distal extremity of the right middle finger. The right hand was kept close to the volunteer's heart with the help of a sling, which fixed the volunteer's arm to his chest throughout the experiment. The signal acquisition frequency was sampled at $1000 \mathrm{~Hz}$.

The extraction of beat-to-beat variability series was carried out according to previous descriptions (20). After extraction of the series, the 256-point sequences with the greatest stability were chosen for both positions (21).

\section{Baroreflex sensitivity}

Baroreflex was evaluated by phase, coherence $\left(\mathrm{K}^{2}\right)$, and gain $(\alpha)$. Baroreflex was calculated by cross-spectral analysis using a bivariate autoregressive model (22). The phase is computed as the phase of the cross-spectrum from $\mathrm{BP}$ to $\mathrm{RR}$ interval $(\mathrm{RRi})$ and represents the delay between the change in BP and the subsequent change in $\mathrm{RRi}$, measured in radians. Coherence $\left(\mathrm{K}^{2}\right)$ was used to estimate the strength of the coupling between $\mathrm{RRi}$ and $\mathrm{BP}$. The squared coherence is computed as the ratio of the squared modulus of the cross-spectrum to the product of the power spectra. In this study, phase and coherence were sampled at the frequency of vasomotor oscillations (Mayer waves) at the low frequency band (LF), which oscillates between $0.04-0.15 \mathrm{~Hz}$ and is related to the sympathetic predominance $(21,23)$. Gain in the LF band was calculated as the square root of the ratio of the LF power of the RRi series to that of the $\mathrm{BP}$ series (24) and characterizes the relation between $\mathrm{BP}$ and RRi.

\section{Sample entropy (SampEn)}

Entropy is a measure of the information needed to predict the future state of a system. It provides a characterization of the dynamics of a signal, the greater the dynamics, the greater the entropy and the less predictable the system $(6,7)$.

Sample entropy (SampEn) (25) is a complexity measure used to quantify regularity of time series, especially short and noisy sequences. It is a measure that monitors how much a set of patterns are close together for a few observations. Lower values for SampEn indicate regularity and predictability. In this study, it was computed with $\mathrm{m}=2$, $r=0.2$ times the standard deviation of the signal, and $n=256$. $M$ represents the length of the vector (patterns) to be compared and $r$ represents the radius within which the comparison between the vectors is achieved (similarity criteria).

\section{Intervention protocol}

The multicomponent exercise intervention was designed considering the recommendations proposed by the American College of Sports Medicine (26). The protocol consisted of aerobic, muscle strength, flexibility, and balance exercises. It was performed during 16 weeks, for three nonconsecutive days, with 60-minute sessions. The sessions were composed by: a) 10 min of warm-up (light walk); b) $20 \mathrm{~min}$ of aerobic exercises; c) $10 \mathrm{~min}$ of balance exercise; d) $15 \mathrm{~min}$ of resistance exercises; and e) 5 min of cool-down. Details concerning exercise type, intensity, and progression are described in a previous study (27).

Before the beginning of the intervention, all volunteers of the MulTI were invited to participate in three sessions on non-consecutive days for familiarization process and determination of resistance training load.

\section{Statistical analysis}

Sample size calculation was based on a previous study (11) that presented cardiovascular parameters as main outcome in prefrail and frail population. The ShapiroWilk test was used to verify the normality of data distribution. The Student's paired $t$-test was used to compare 
age, anthropometric characteristics (weight, stature, BMI), number of comorbidities, educational level, MMSE total score, and SPPB total score at baseline. The chi-squared test was applied to compare sex and each frailty criteria according to phenotype.

A linear mixed model test was used to assess the effect of the training at baseline and post-intervention, between MulTI and CG on functional measures (walk distance and gait speed) and on cardiovascular variables in supine rest position. The same test was used to assess the effect on postural maneuver separately for each group, considering assessments (baseline $\times$ post-intervention) and positions (supine $\times$ orthostatic) in cardiovascular variables.

The significance level established was $5 \%$. Statistical analysis was performed using the software IBM SPSS Statistics, version 20.0 (USA).

\section{Results}

The volunteers' age, and anthropometric and clinical characteristics are presented in Table 1. There was no difference in sex, age, weight, stature, number of comorbidities, educational level, MMSE total score, and frailty criteria between the groups. There was significant difference for BMI $(P=0.032)$ and SPPB total score $(P=0.006)$ between the groups.

Table 2 presents BRS and SampEn results in rest supine position. In terms of BRS, there was no difference in any parameter. Concerning SampEn, despite groups, there was a significant reduction in post-assessment compared to baseline $(P=0.036)$.
The postural maneuver behavior of each group between assessments is reported in Tables 3 and 4 . Thus, the effects of positions (supine $\times$ orthostatic) and assessments (baseline $\times$ post-intervention) were tested for each group considering BRS and SampEn. The CG did not present significant differences for position, assessment, or interaction in BRS parameters. Nonetheless, there was a reduction in post-intervention assessment related to baseline for SampEn $(P=0.008)$ (Table 3). The MulTI group did not present any significant differences for BRS or SampEn values between position, assessments, or their interaction (Table 4).

Considering the functional measures, there was a group effect for walk distance $(P=0.019)$ indicating the $C G$ presented higher values compared to MulTI. Furthermore, CG also presented higher values for gait speed than MulTI $(P<0.001)$ and in post-intervention assessment both groups presented a better performance (increase) compared to baseline $(P=0.001)$ (Table 5).

\section{Discussion}

Complexity is an elusive concept, which has been inserted in biological and health studies once physiological systems are featured by a dynamic network of multiple interacting inputs between control mechanisms (7). Physiological complexity is directly related to an adaptive capacity of the organism to ever-changing environment (28). Thus, a healthy organism is characterized by presence of adaptability properties, which allows effective coping and high functionality to respond to unpredictable stimuli and stresses of daily life $(7,29)$.

Table 1. Age, anthropometric, and clinical characteristics.

\begin{tabular}{lcrl}
\hline & MulTI $(\mathrm{n}=12)$ & \multicolumn{1}{c}{ CG $(\mathrm{n}=9)$} & P-value \\
\hline Female gender, $\mathrm{n}(\%)$ & $10(83.3)$ & $5(55.6)$ & 0.163 \\
Age (years) & $77.00 \pm 6.80$ & $73.78 \pm 6.28$ & 0.281 \\
Weight $(\mathrm{kg})$ & $76.26 \pm 12.72$ & $68.54 \pm 8.69$ & 0.135 \\
Stature $(\mathrm{cm})$ & $154.83 \pm 6.48$ & $159.56 \pm 5.90$ & 0.102 \\
BMI $\left(\mathrm{kg} / \mathrm{m}^{2}\right)$ & $31.95 \pm 5.47$ & $27.02 \pm 3.74$ & $\mathbf{0 . 0 3 2}$ \\
Comorbidities & $2.33 \pm 1.30$ & $2.44 \pm 1.81$ & 0.872 \\
Educational level (years) & $4.42 \pm 2.57$ & $6.83 \pm 4.37$ & 0.207 \\
MMSE total score & $24.50 \pm 2.54$ & $25.89 \pm 3.95$ & 0.339 \\
SPPB total score & $7.58 \pm 1.31$ & $9.44 \pm 1.42$ & $\mathbf{0 . 0 0 6}$ \\
Frailty criteria & & & \\
$\quad$ Unintentional weight loss, $\mathrm{n}(\%)$ & $3(25.00)$ & $2(22.22)$ & 0.647 \\
$\quad$ Low activity level, $\mathrm{n}(\%)$ & $3(25.00)$ & $3(33.33)$ & 0.523 \\
Weakness, $\mathrm{n}(\%)$ & $3(25.00)$ & $2(22.22)$ & 0.647 \\
Exhaustion, $\mathrm{n}(\%)$ & $6(50.00)$ & $4(44.44)$ & 0.575 \\
Slowness, $\mathrm{n}(\%)$ & $2(16.66)$ & $0(0.00)$ & 0.314 \\
\hline
\end{tabular}

Data are reported as means $\pm S D$ or total of individuals (percent). MulTI: multicomponent training intervention; $C G$ : control group; BMI: body mass index; MMSE: Mini Mental State Examination; SPPB: Short Physical Performance Battery. $\mathrm{P}<0.05$ compared to $C G$ (Student's paired $t$-test and chi-squared test). 
Table 2. SampEn and baroreflex sensitivity in MulTI and CG groups in supine position.

\begin{tabular}{|c|c|c|c|c|c|c|c|}
\hline & \multicolumn{2}{|c|}{ MulTI } & \multicolumn{2}{|c|}{ CG } & \multicolumn{3}{|c|}{ P-value } \\
\hline & Baseline & Post & Baseline & Post & Groups & Assessments & Interaction \\
\hline SampEn & $1.72 \pm 0.58$ & $1.30 \pm 0.46$ & $1.72 \pm 0.38$ & $1.32 \pm 0.43$ & 0.971 & $0.036^{*}$ & 0.945 \\
\hline \multicolumn{8}{|l|}{ BRS } \\
\hline Coherence & $0.51 \pm 0.12$ & $0.36 \pm 0.16$ & $0.51 \pm 0.17$ & $0.55 \pm 0.28$ & 0.992 & 0.076 & 0.117 \\
\hline Phase (rad) & $-1.10 \pm 1.19$ & $-1.76 \pm 1.09$ & $-1.16 \pm 1.61$ & $-0.81 \pm 2.59$ & 0.830 & 0.573 & 0.889 \\
\hline Gain $(\mathrm{ms} / \mathrm{mmHg})$ & $5.03 \pm 3.59$ & $7.07 \pm 4.00$ & $3.47 \pm 1.45$ & $7.43 \pm 5.84$ & 0.521 & 0.239 & 0.727 \\
\hline
\end{tabular}

Data are reported as means \pm SD. SampEn: Sample entropy; BRS: baroreflex sensitivity; MulTI: multicomponent training intervention; CG: control group. ${ }^{*} \mathrm{P}<0.05$ compared to baseline (baseline $>$ post, linear mixed model test).

Table 3. SampEn and baroreflex sensitivity (BRS) in control group.

\begin{tabular}{|c|c|c|c|c|c|c|c|}
\hline & \multicolumn{2}{|c|}{ Baseline } & \multicolumn{2}{|c|}{ Post } & \multicolumn{3}{|c|}{ P-value } \\
\hline & Supine & Orthostatic & Supine & Orthostatic & Position & Assessments & Interaction \\
\hline SampEn & $1.72 \pm 0.38$ & $1.75 \pm 0.50$ & $1.32 \pm 0.43$ & $1.06 \pm 0.33$ & 0.880 & $0.008^{*}$ & 0.366 \\
\hline \multicolumn{8}{|l|}{ BRS } \\
\hline Coherence & $0.51 \pm 0.17$ & $0.50 \pm 0.22$ & $0.55 \pm 0.28$ & $0.58 \pm 0.23$ & 0.942 & 0.314 & 0.719 \\
\hline Phase (rad) & $-1.16 \pm 1.61$ & $-1.77 \pm 0.84$ & $-0.81 \pm 2.59$ & $-1.34 \pm 0.47$ & 0.410 & 0.615 & 0.966 \\
\hline Gain (ms/mmHg) & $3.47 \pm 1.45$ & $4.14 \pm 2.70$ & $7.43 \pm 5.84$ & $4.23 \pm 2.99$ & 0.684 & 0.932 & 0.082 \\
\hline
\end{tabular}

Data are reported as means \pm SD. SampEn: sample entropy. ${ }^{*} \mathrm{P}<0.05$ compared to baseline (baseline $>$ post, linear mixed model test).

Table 4. SampEn and baroreflex sensitivity (BRS) in multicomponent training intervention group.

\begin{tabular}{|c|c|c|c|c|c|c|c|}
\hline & \multicolumn{2}{|c|}{ Baseline } & \multicolumn{2}{|c|}{ Post } & \multicolumn{3}{|c|}{ P-value } \\
\hline & Supine & Orthostatic & Supine & Orthostatic & Position & Assessments & Interaction \\
\hline SampEn & $1.72 \pm 0.58$ & $1.53 \pm 0.43$ & $1.30 \pm 0.46$ & $1.36 \pm 0.42$ & 0.320 & 0.231 & 0.316 \\
\hline \multicolumn{8}{|l|}{ BRS } \\
\hline Coherence & $0.51 \pm 0.12$ & $0.49 \pm 0.13$ & $0.36 \pm 0.16$ & $0.46 \pm 0.19$ & 0.730 & 0.593 & 0.152 \\
\hline Phase (rad) & $-1.10 \pm 1.19$ & $-1.87 \pm 0.89$ & $-1.76 \pm 1.09$ & $-1.49 \pm 1.34$ & 0.106 & 0.385 & 0.090 \\
\hline Gain $(\mathrm{ms} / \mathrm{mmHg})$ & $5.03 \pm 3.59$ & $2.76 \pm 1.99$ & $7.07 \pm 4.00$ & $4.01 \pm 4.65$ & 0.133 & 0.449 & 0.713 \\
\hline
\end{tabular}

Data are reported as means \pm SD. SampEn: sample entropy. Linear mixed model test.

Table 5. Functional measures of the multicomponent training intervention group (MulTI) and control group (CG).

\begin{tabular}{|c|c|c|c|c|c|c|c|}
\hline \multirow[t]{2}{*}{ Functional measures } & \multicolumn{2}{|c|}{ MulTI } & \multicolumn{2}{|c|}{ CG } & \multicolumn{3}{|c|}{ P-value } \\
\hline & Baseline & Post & Baseline & Post & Groups & Assessments & Interaction \\
\hline Walk distance (6MWT) $(\mathrm{m})$ & $326.42 \pm 64.42$ & $323.50 \pm 83.41$ & $401.00 \pm 53.18$ & $414.00 \pm 58.42$ & $0.019^{*}$ & 0.741 & 0.375 \\
\hline Gait speed (m/s) & $0.74 \pm 0.20$ & $0.91 \pm 0.16$ & $1.05 \pm 0.12$ & $1.11 \pm 0.14$ & $<0.001^{*}$ & $0.001^{*}$ & 0.183 \\
\hline
\end{tabular}

Data are reported as means $\pm S D$. $6 \mathrm{MWT}$ : six-minute walk test. ${ }^{*} \mathrm{P}<0.05$ compared to baseline (post $>$ baseline, linear mixed model test) and between groups (MulTI vs CG, linear mixed model test).

With the aging process, the number and connectedness of these inputs is reduced and the output signal is simplified, which limits responses to stressors and features as a reduced physiological complexity (7). As complexity falls further, it may impair functional capacity until crossing the frailty threshold (7), resulting in evident vulnerability to adverse outcomes (4). Therefore, the greater the number of dysregulated physiological systems, the stronger the likelihood of frailty development (30). 
Previous studies identified impairment in cardiovascular control assessed by complexity measurements in frail older adults $(7,10,11)$. On the other hand, it is unclear if this impairment could be present in prefrail older adults, even in a lesser proportion. Structural and functional alterations of noninvasive biomarkers of cardiovascular disease (CVD) such as level of carotid stenosis and wall thickness are prevalent in frail as well as in prefrail individuals (31). Also, the negative influence of CVD in HR complexity has already been demonstrated (32). According to our findings, HR complexity is impaired in prefrail older adults even in rest supine condition, once a significant reduction was detected in the post-assessment compared to baseline, despite the group.

Once the network structure of the physiological system enables alternate pathways to be used to achieve the same functions, even in adverse conditions as aging or disease, the organism may keep functional capacity if other neural components and their connections could compensate (7). Nevertheless, in frailty course probably there is a limited response repertoire due to lesser interaction among the physiological systems; consequently, the individual may present a too succinct/ insufficient or exacerbated response. An example of this is the orthostatic hypotension described in frail individuals (33). Considering that the baroreflex represents an interaction among control subsystems responsible for BP homeostasis (34), in agreement with a previous study (12), our data suggested there is also an impairment in BRS in prefrail individuals.

The active postural maneuver is a functional task that triggers some physiological alterations in cardiac contractility, vasoconstriction, and HR by increasing in sympathetic modulation and vagal withdrawn $(35,36)$. Thus, it is expected that the baroreflex mechanism acts by a fast increase in HR and BP dropping until it restores to adequate levels. Concerning the cardiovascular dynamics response to postural maneuver, a healthy organism presents a decrease in gain of BRS $(35,37)$, increase in $\mathrm{K}^{2}$ values (37), as well a decrease in HR complexity (9). In the frailty course, it seems the mechanisms fail and the response is impaired, as shown by the performance of prefrail individuals in the present study once both groups did not respond adequately.

It remains unknown if complexity of cardiovascular control could be restored by any kind of intervention. To date, few studies have been developed aiming to investigate the effect of exercise intervention on cardiovascular complexity and BRS. Resistance training conducted among hypertensive older adults and young individuals promoted an increase in HR complexity, whereas no change was identified in traditional measurements of $\operatorname{HR}(15,16)$. Concerning endurance training, it was demonstrated that a 21-week progressive program was more effective in improvement (increase) of HR complexity in middle-aged women compared to combined strength and endurance training or strength training alone (38). Similarly, 4-week endurance training in hypertensive middle-aged individuals improved BRS while the strength training had the opposite effect (17).

The cardiovascular benefits from distinct exercise training are divergent and the underlying mechanisms are still uncertain. It has been suggested that physical exercise, especially endurance and resistance training, could potentially restore at least partially the complex dynamics in physiological systems (5) through the development of new network connections as well as a reorganization of information outflow $(5,7)$ and, consequently, improve functional health.

Currently, there are few intervention studies destined to physiological complexity outcomes. It has been assumed that a protocol design that targets multi-systems effects and treats risk factors of disability may have the greatest potential to restore healthy dynamics in biological systems (5). In consonance, multicomponent exercise training has been considered as the most adequate intervention modality to frailty management especially in earlier stages $(13,14)$.

In this sense, it was hypothesized that the multicomponent intervention protocol developed in the present study would be capable to improve the dynamics evolved in cardiovascular control. On the assumption that frailty is featured by multisystem dysregulation (30), it was thought a broad approach could mutually affect multiple physiological "gears" and restore their interaction, reflecting in HR complexity as well BRS. Nonetheless, our findings indicated this intervention did not improve these parameters. It is possible the protocol design (load, duration, and/or progression) was not the most adequate.

Some authors argue that although an intuitive rationality guides multicomponent approaches, the complex systems theory suggests the modification of a single component of a system may contribute to global (holistic) effects on system behavior (39). This seems to be consistent with theoretical basis that considers the frailty progression as dependent at least to one abnormal system to be able to trigger a downward spiraling and affect other healthy functional systems until achieving a whole dysregulated state (30).

Related to the functional measures, there was no effect of multicomponent training in walk distance and gait speed in prefrail individuals. At baseline and even post-intervention, CG presented better performance (greater walk distance and faster gait speed) compared to MulTI. The aerobic load developed in the multicomponent intervention (60 min/week) might not be enough to promote benefits. According to the American College of Sports Medicine (26), at least $150 \mathrm{~min} /$ week of moderate-intensity aerobic exercise has been suggested for older adults.

Lastly, gait speed has been considered as a prognostic predictive factor for all-cause mortality in older 
patients with cardiovascular disease (40), and also complexity indexes have already proven their prognostic value in some pathological conditions as coronary artery disease (32). Thus, usage of metrics to quantify complexity in addition to functional measurements may contribute to the recommendations designated to specific programs for prefrail individuals and be a potential method of risk stratification for this population (8).

\section{Conclusions}

Prefrail individuals demonstrated a reduction in HR complexity in rest condition, which confirms impairment in the autonomic nervous system related to cardiovascular control even in intermediate frailty stage. Concerning postural maneuver, they did not present the expected response, suggesting a difficulty to deal with provocative tasks that affect homeostasis. Furthermore, the 16-week multicomponent exercise training did not improve HR complexity, BRS, and functional measures (walk distance and gait speed).

Frailty management is a challenge because it presents specificities related to wide and multidirectional physiological features. In this sense, the earliest identification of

\section{References}

1. Dent E, Kowal P, Hoogendijk EO. Frailty measurement in research and clinical practice: a review. Eur J Intern Med 2016; 31: 3-10, doi: 10.1016/j.ejim.2016.03.007.

2. Morley JE, Vellas B, van Kan GA, Anker SD, Bauer JM, Bernabei $\mathrm{R}$, et al. Frailty consensus: a call to action. $J \mathrm{Am}$ Med Dir Assoc 2013; 14: 392-397, doi: 10.1016/j.jamda. 2013.03.022.

3. Clegg A, Young J, lliff S, Rikkert MO, Rockwood K. Frailty in elderly people. Lancet 2013; 381: 752-762, doi: 10.1016/ S0140-6736(12)62167-9.

4. Fried LP, Tangen CM, Walston J, Newman AB, Hirsch C, Gottdiener J, et al. Frailty in older adults: evidence for a phenotype. J Gerontol A Biol Sci Med Sci 2001; 56: M146M156, doi: 10.1093/gerona/56.3.M146.

5. Lipsitz LA. Physiological complexity, aging, and the path to frailty. Sci Aging Knowledge Environ 2004; 2004: pe16, doi: 10.1126/sageke.2004.16.pe16.

6. Lipsitz LA, Goldberger AL. Loss of 'complexity' and aging. Potential applications of fractals and chaos theory to senescence. JAMA 1992; 267: 1806-1809, doi: 10.1001/jama. 1992.03480130122036.

7. Lipsitz LA. Dynamics of stability: the physiologic basis of functional health and frailty. J Gerontol A Biol Sci Med Sci 2002; 57: B115-B125, doi: 10.1093/gerona/57.3.B115.

8. Walston J, Hadley EC, Ferrucci L, Guralnik JM, Newman $A B$, Studenski SA, et al. Research agenda for frailty in older adults: toward a better understanding of physiology and etiology: summary from the American geriatrics society/ national institute on aging research conference on frailty in older adults. J Am Geriatr Soc 2006; 54: 991-1001, doi: 10.1111/j.1532-5415.2006.00745.x. systemic deficits through sensitive tools may help in the development of effective interventions targeted to prefrail individuals. Future studies should be conducted testing the efficacy of different types of training on cardiovascular dynamics and also on functional measures in a large sample.

\section{Study limitations}

The cardiopulmonary exercise testing, which is considered the gold standard tool for determination of maximal oxygen uptake $\left(\mathrm{VO}_{2}\right.$ max), was not performed to assess aerobic capacity.

\section{Acknowledgments}

This work was supported by Coordination for the Improvement of Higher Education Personnel (CAPES) (\#88882.332817/2014-01 to M.S.S. Buto). All the authors are grateful to "Programa de Pós-Graduação em Fisioterapia, Universidade Federal de São Carlos" (PPGFT, UFSCar) for providing the infrastructure to develop this study.

9. Porta A, Faes L, Bari V, Marchi A, Bassani T, Nollo G, et al. Effect of age on complexity and causality of the cardiovascular control: comparison between model-based and modelfree approaches. PLoS One 2014; 9: e89463, doi: 10.1371/ journal.pone.0089463.

10. Chaves PHM, Varadhan R, Lipsitz LA, Stein PK, Windham BG, Tian J, et al. Physiological complexity underlying heart rate dynamics and frailty status in community-dwelling older women. J AM Geriatr Soc 2008; 56: 1698-1703, doi: 10.1111/j.1532-5415.2008.01858.x.

11. Katayama PL, Dias DPM, Silva LEV, Virtuoso-Junior JS, Marocolo M. Cardiac autonomic modulation in non-frail, prefrail and frail elderly women: a pilot study. Aging Clin Exp Res 2015; 27: 621-629, doi: 10.1007/s40520-015-0320-9.

12. Buto MSS, Catai AM, Vassimon-Barroso V, Gois MO, Porta A, Takahashi ACM. Baroreflex sensitivity in Frailty Syndrome. Braz J Med Biol Res 2019; 52: e8079, doi: 10.1590/ 1414-431x20198079.

13. Dedeyne L, Deschodt M, Verschueren S, Tournoy J, Gielen $E$. Effects of multi-domain interventions in (pre)frail elderly on frailty, functional, and cognitive status: a systematic review. Clin Interv Aging 2017; 12: 873-896, doi: $10.2147 /$ CIA.S130794.

14. Jadcazk AD, Makwana N, Luscombe-Marsh N, Visvanathan $R$, Schultz TJ. Effectiveness of exercise interventions on physical function in community-dwelling frail older people: an umbrella review of systematic reviews. JBI Database System Rev Implement Rep 2018; 16: 752-775, doi: 10.11124/JBISRIR-2017-003551.

15. Heffernan KS, Fahs CA, Shinsako KK, Jae SY, Fernhall B. Heart rate recovery and heart rate complexity following 
resistance exercise training and detraining in young men. Am J Physiol Heart Circ Physiol 2007; 293: H3180-H3186, doi: 10.1152/ajpheart.00648.2007.

16. Millar PJ, Levy AS, McGowan CL, McCartney N, MacDonald MJ. Isometric handgrip training lowers blood pressure and increases heart rate complexity in medicated hypertensive patients. Scand J Med Sci Sports 2013; 23: 620-626, doi: 10.1111/j.1600-0838.2011.01435.x.

17. Collier SR, Kanaley JA, Carhart Jr R, Frechette V, Tobin MM, Bennett N, et al. Cardiac autonomic function and baroreflex changes following 4 weeks of resistance versus aerobic training in individuals with pre-hypertension. Acta Physiol (Oxf) 2009; 195: 339-348, doi: 10.1111/j.1748-1716.2008. 01897.x

18. Wayne PM, Manor B, Novak V, Costa MD, Hausdorff JM, Goldberger AL, et al. A systems biology approach to studying Tai Chi, physiological complexity and healthy aging: design and rationale of a pragmatic randomized controlled trial. Contemp Clin Trials 2013; 34: 21-34, doi: 10.1016/j.cct.2012.09.006

19. Souza A, Nery CAS, Marciano LHSC, Garbino JA. Avaliação da neuropatia periférica: correlação entre a sensibilidade cutânea dos pés, achados clínicos e eletroneuromiográficos. Acta Fisiatr 2005; 12: 87-93, doi: 10.11606/ issn.2317-0190.v12i3a102530.

20. Faes L, Nollo G, Porta A. Mechanisms of causal interaction between short-term RR interval and systolic arterial pressure oscillations during orthostatic challenge. J Appl Physiol (1985) 2013; 114: 1657-1667, doi: 10.1152/japplphysiol. 01172.2012.

21. Task Force of the European Society of Cardiology and the North American Society of Pacing and Electrophysiology. Heart rate variability: standards of measurement, physiological interpretation and clinical use. Circulation 1996; 93: 1043-1065, doi: 10.1161/01.CIR.93.5.1043.

22. Porta A, Aletti F, Vallais F, Baselli G. Multimodal signal processing for the analysis of cardiovascular variability. Philos Trans A Math Phys Eng Sci 2009; 367: 391-409, doi: $10.1098 /$ rsta.2008.0229.

23. Pagani M, Lombardi F, Guzzetti S, Rimoldi O, Furlan R, Pizzinelli $P$, et al. Power spectral analysis of heart rate and arterial pressure variabilities as a marker of sympatho-vagal interaction in men and conscious dog. Circ Res 1986; 59: 178-193, doi: 10.1161/01.RES.59.2.178.

24. Nollo G, Porta A, Faes L, Del Greco M, Disertori M, Ravelli F. Causal linear parametric model for baroreflex gain assessment in patients with recent myocardial infarction. $A m \mathrm{~J}$ Physiol Heart Circ Physiol 2001; 280: H1830-H1839, doi: 10.1152/ajpheart.2001.280.4.H1830.

25. Richman JS, Moorman JR. Physiological time-series analysis using approximate entropy and sample entropy. Am J Physiol Heart Circ Physiol 2000; 278: H2039-H2049, doi: 10.1152/ajpheart.2000.278.6. H2039.

26. American College of Sports Medicine, Chodzko-Zajko WJ, Proctor DN, Singh MAF, Minson CT, Nigg CR, et al. American College of Sports Medicine position stand. Exercise and physical activity for older adults. Med Sci Sports Exerc 2009; 41: 1510-1530, doi: 10.1249/MSS.0b013e3181a0c95c.

27. Buto MSS, Fiogbé E, Vassimon-Barroso V, Rossi PG, Farche ACS, Carnavale BF, et al. Pre-frail multicomponent training intervention project for complexity of biological signals, functional capacity and cognition improvement in pre-frail older adults: a blinded randomized controlled study protocol. Geriatr Gerontol Int 2019; 19: 684-689, doi: 10.1111/ggi.13672.

28. Javorka M, Krohova J, Czippelova B, Turianikova Z, Lazarova Z, Wiszt R, et al. Towards understanding the complexity of cardiovascular oscillations: insights from information theory. Comput Biol Med 2018; 98: 48-57, doi: 10.1016/j.compbiomed.2018.05.007.

29. Goldberger AL, Amaral LAN, Hausdorff JM, Ivanov PCH, Peng CK, Stanley HE. Fractal dynamics in physiology: Alterations with disease and aging. Proc Natl Acad Sci USA 2002; 99: 2466-2472, doi: 10.1073/pnas.012579499.

30. Fried LP, Xue QL, Cappola AR, Ferrucci L, Chaves P, Varadhan $R$, et al. Nonlinear multisystem physiological dysregulation associated with frailty in older women: implications for etiology and treatment. J Gerontol A Biol Sci Med Sci 2009; 64: 1049-1057, doi: 10.1093/gerona/glp076.

31. Newman AB, Gottdiener JS, McBurnie MA, Hirsch CH, Kop WJ, Tracy R, et al. Associations of subclinical cardiovascular disease with Frailty. J Gerontol A Biol Sci Med Sci 2001; 56: M158-M166, doi: 10.1093/gerona/56.3.M158.

32. Chen $C$, Jin $Y$, Lo IL, Zhao $H$, Sun B, Zhao Q, et al. Complexity change in cardiovascular disease. Int J Biol Sci 2017; 13: 1320-1328, doi: 10.7150/ijbs.19462.

33. Rockwood MRH, Howlett SE, Rockwood K. Orthostatic hypotension $(\mathrm{OH})$ and mortality in relation to age, blood pressure and frailty. Arch Gerontol Geriatr 2012; 54: e255e260, doi: 10.1016/j.archger.2011.12.009.

34. Di Rienzo M, Parati G, Radaelli A, Castiglioni P. Baroreflex contribution to blood pressure and heart rate oscillations: time scales, time-variant characteristics and nonlinearities. Philos Trans A Math Phys Eng Sci 2009; 367: 1301-1318, doi: 10.1098/rsta.2008.0274.

35. Laitinen T, Niskanen L, Geelen G, Länsimies E, Hartikainen J. Age dependency of cardiovascular autonomic responses to head-up tilt in healthy subjects. J Appl Physiol 2004; 96: 2333-2340, doi: 10.1152/japplphysiol.00444.2003.

36. Monahan KD. Effect of aging on baroreflex function in humans. Am J Physiol Regul Integr Comp Physiol 2007; 293: R3-R12, doi: 10.1152/ajpregu.00031.2007.

37. Porta A, Castiglioni P, Di Rienzo M, Bari V, Bassani T, Marchi $A$, et al. Information domain analysis of the spontaneous baroreflex during pharmacological challenges. Auton Neurosci 2013; 178: 67-75, doi: 10.1016/j.autneu.2013.03.003.

38. Karavirta L, Costa MD, Goldberger AL, Tulppo MP, Laaksonen DE, Nyman K, et al. Heart rate dynamics after combined strength and endurance training in middle-aged women: heterogeneity of responses. PLoS One 2013; 8: e72664, doi: 10.1371/journal.pone.0072664.

39. Manor B, Lipitz LA. Physiologic complexity and aging: Implications for physical function and rehabilitation. Prog Neuropsychopharmacol Biol Psychiatry 2013; 45: 287-293, doi: 10.1016/j.pnpbp.2012.08.020.

40. Kamiya K, Hamazaki N, Matsue $\mathrm{Y}$, Mezzani A, Corrà U, Matsuzawa R, et al. Gait speed has comparable prognostic capability to six-minute walk distance in older patients with cardiovascular disease. Eur J Prev Cardiol 2018; 25: 212219, doi: $10.1177 / 2047487317735715$. 